\title{
Sestrin 2 Attenuates Rat Hepatic Stellate Cell (HSC) Activation and Liver Fibrosis via an mTOR/AMPK-Dependent Mechanism
}

\author{
Yi-Bing $\mathrm{Hu}^{\mathrm{a}, \mathrm{b}} \quad$ Xiao-Ting $\mathrm{Ye}^{\mathrm{b}}$ Qing-Qing Zhou ${ }^{\mathrm{b}}$ Rong-Quan Fu \\ aDepartment of Gastroenterology, Jinhua Hospital of Zhejiang University, Jinhua, bDepartment of \\ Infectious Diseases, The Third Affiliated Hospital of Wenzhou Medical University, Rui'an, China
}

\section{Key Words}

Liver fibrosis $•$ Sestrin $2 \cdot$ Hepatic stellate cells $•$ AMPK $・$ mTOR

\begin{abstract}
Background/Aims: Sestrin 2 is associated with the pathophysiology of several diseases. The aim of this study was to investigate the effects and potential mechanisms of Sestrin 2 in rat hepatic stellate cells (HSCs) during liver fibrogenesis. Methods: In this study, Sestrin 2 protein expression was detected in rat HSC-T6 cells challenged with transforming growth factor- $\beta$ (TGF- $\beta$ ) and in mice treated with carbon tetrachloride $\left(\mathrm{CCl}_{4}\right)$, a well-known model of hepatic fibrosis. Next, HSC-T6 cells and fibrotic mice were transfected with lentivirus. The mRNA expression levels of markers of liver fibrosis [alpha-smooth muscle actin ( $\alpha$-SMA) and collagen $1 \mathrm{~A} 1(\mathrm{Col1A} 1)]$ were analyzed by quantitative reverse transcription-polymerase chain reaction (RT-PCR). Cell death and proliferation were evaluated by the MTT assay, and biochemical markers of liver damage in serum [alanine transaminase (ALT) and aspartate transaminase (AST)] were also measured using a biochemical analyzer. Histopathological examination was used to evaluate the degree of liver fibrosis, and protein expression [phospho-adenosine monophosphate-activated protein kinase (p-AMPK), AMPK, phospho-mammalian target of rapamycin (p-mTOR), and mTOR] was determined by western blotting. Results: We found that Sestrin 2 was elevated in both the HSC-T6 cell and hepatic fibrosis models. In vitro, overexpression of Sestrin 2 attenuated the mRNA levels of $\alpha$-SMA and Col1A1, suppressed $\alpha$-SMA protein expression, and modulated HSC-T6 cell proliferation. In vivo, overexpression of Sestrin 2 reduced the ALT and AST levels as well as the $\alpha-S M A$ and Col1A1 protein expression in the $\mathrm{CCl}_{4}$ model of liver fibrosis. Moreover, the degree of liver fibrosis was ameliorated. Interestingly, overexpression of Sestrin 2 increased p-AMPK but decreased p-mTOR protein expression. Conclusion: Our findings indicate that Sestrin 2 may attenuate the activation of HSCs and ameliorate liver fibrosis, most likely via upregulation of AMPK phosphorylation and suppression of the mTOR signaling pathway.




\section{Introduction}

Liver fibrosis occurs as a result of chronic hepatocellular injury due to various etiologies, including viral hepatitis, nonalcoholic steatohepatitis, and autoimmune hepatitis [1-3]. Persistent hepatocyte damage induces the release of cytokines and growth factors, such as transforming growth factor- $\beta$ (TGF- $\beta$ ), which leads to the activation of hepatic stellate cells (HSCs) [4]. The transformation of quiescent HSCs into activated myofibroblasts results in the upregulation of alpha-smooth muscle actin ( $\alpha$-SMA) and the deposition of extracellular matrix, which play a pivotal role in the occurrence and development of liver fibrosis [5]. As liver fibrosis progresses, patients may suffer from liver dysfunction and portal hypertension [6]. Therefore, a better understanding of the potential mechanisms involved in liver fibrosis is required.

Sestrins are a family of stress-inducible proteins that are highly conserved among mammals. Sestrins consist of Sestrin 1, Sestrin 2, and Sestrin $3[7,8]$. In response to oxidative stress, Sestrin 2 is induced to regulate cell viability, growth, and metabolism [9, 10]. The expression of Sestrin 2 is associated with several stress conditions and, in turn, controls metabolic homeostasis as well as prevents relevant pathological conditions [11]. Sestrin 2 also functions as a tumor inhibitor that can suppress autophagy [8]. In antioxidant defense mechanisms, induction of Sestrin 2 plays a crucial part in regulating the adenosine monophosphate-activated protein kinase (AMPK)-mammalian target of rapamycin (mTOR) signaling pathway [12-15]. However, the effect of Sestrin 2 in liver fibrogenesis remains unknown. Moreover, the roles of AMPK stimulation and its subsequent suppression of mTOR activity are not clear $[16,17]$.

In this work, we investigated the impact of Sestrin 2 in the carbon tetrachloride $\left(\mathrm{CCl}_{4}\right)$ induced hepatic fibrosis mouse model and in rat HSCs. We also studied the related underlying mechanism. Interestingly, we found a novel role of Sestrin 2 in the modulation of the AMPKmTOR signaling pathway, thus providing a new perspective and direction for the treatment of liver fibrosis.

\section{Materials and Methods}

\section{Animal procedure}

The protocols for this animal study were approved by the Committee of Animal Care and Use at Wenzhou Medical University. Male C57BL/6 mice (8 weeks old), weighing 18-22 g, were purchased from the Shanghai Laboratory Center (Shanghai, China) and acclimatized for 7 days. Mice were fed with water and standard food and were housed at $20 \pm 2{ }^{\circ} \mathrm{C}$ with a $12-12 \mathrm{~h} \mathrm{light/dark} \mathrm{cycle} \mathrm{under} \mathrm{specific} \mathrm{pathogen-free}$ conditions. Mice were treated with an intraperitoneal injection of $\mathrm{CCl}_{4}(0.6 \mathrm{~mL} / 100 \mathrm{~g}$, body weight $)$ diluted in olive oil ( $2: 3 ; \mathrm{v} / \mathrm{v})$, twice a week, for 8 weeks. The vehicle group included mice that received only olive oil at the same time points. At $48 \mathrm{~h}$ after the last $\mathrm{CCl}_{4}$ injection, the mice were killed under anesthesia, and the liver tissues and blood samples were collected for subsequent analyses.

\section{Cell culture}

The HSC-T6 cell line, an immortalized rat HSC line, was grown in a 5\% $\mathrm{CO}_{2}$-humidified incubator at $37^{\circ} \mathrm{C}$ in high-glucose Dulbecco's modified Eagle's medium (Gibco, USA), supplemented with 10\% fetal bovine serum (Gibco), $100 \mathrm{U} / \mathrm{mL}$ penicillin, and $100 \mathrm{U} / \mathrm{mL}$ streptomycin. The culture medium was replaced every 2 days.

\section{Lentiviral transfection}

The lentiviral vector was constructed by Shanghai GenePharma Co., Ltd. (Shanghai, China). Cells were seeded in 6-well plates or 96-well plates and transfected with vector expressing Sestrin 2 (Lenti-Sestrin 2) or empty vector (Lenti-NC), according to the manufacturer's instructions. A multiplicity of infection of 50 was used. Lenti-Sestrin 2 or Lenti-NC was injected into the tail vein once a day $\left(1 \times 10^{9}\right.$ units/100 $\left.\mu \mathrm{L}\right)$. The mice were divided into four groups: (I) Control group ( $\mathrm{n}=8$ ); (II) $\mathrm{CCl}_{4}$ model group ( $\mathrm{n}=8$ ); (III) $\mathrm{CCl}_{4}$ and 


\section{Cellular Physiology Cell Physiol Biochem 2018;51:2111-2122

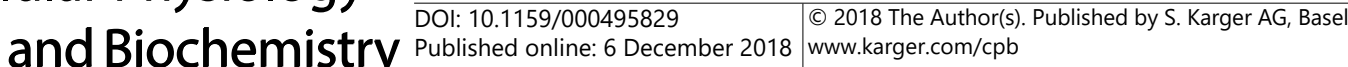

Hu et al.: Sestrin 2 Attenuates Liver Fibrogenesis

Lenti-NC injection group ( $\mathrm{n}=8$ ); and (IV) $\mathrm{CCl}_{4}$ and Lenti-Sestrin 2 injection group ( $\left.\mathrm{n}=8\right)$. After another 4 weeks, the mice were sacrificed for analysis.

\section{Cell proliferation assay}

Approximately $1 \times 10^{4}$ HSC-T6 cells per well were seeded into 96-well plates and divided into one of three groups: (I) Control group; (II) Lenti-NC group; and (III) Lenti-Sestrin 2 group. Forty-eight hours later, $20 \mu \mathrm{L}$ of 3-(4, 5-dimethylthiazol-2-yl)-2, 5-diphenyltetrazolium bromide (MTT) (KeyGEN, China) was added to each well. After $4 \mathrm{~h}$, the mixture was removed and $150 \mu \mathrm{L}$ of dimethyl sulfoxide was added to each well to dissolve the dye for $30 \mathrm{~min}$. The absorbance was measured at $570 \mathrm{~nm}$. The experiments were repeated three times.

\section{Blood biochemistry analysis}

At the time of sacrifice, blood samples were collected from the eyes and centrifuged for $10 \mathrm{~min}$. The serum alanine transaminase (ALT) and aspartate transaminase (AST) levels were determined at the Third Affiliated Hospital using a fully automatic biochemical analyzer (AU5800, Beckman Coulter, USA).

\section{Histological examination of liver}

Liver tissues were harvested after the mice were sacrificed. After fixing with $4 \%$ paraformaldehyde, the tissues were embedded in paraffin and sectioned $(5 \mu \mathrm{m})$. The slices were processed and stained with hematoxylin and eosin (H\&E) for histopathological observation or with Masson's trichrome staining to detect collagen deposition. Immunohistochemical staining of $\alpha$-SMA and collagen 1A1 (Col1A1) was performed on randomly selected sections, which were photographed by light microscopy.

\section{Immunofluorescence staining}

For immunofluorescence staining, HSCs were seeded in 6-well plates and fixed with 4\% paraformaldehyde for $10 \mathrm{~min}$. Then, the cells were blocked with $5 \%$ bovine serum albumin (BSA)/ phosphate-buffered saline for $1 \mathrm{~h}$, followed by incubation with the indicated primary antibody $\alpha$-SMA (Abcam) at $4 \stackrel{\circ}{\circ}$ overnight. Next, secondary antibody (Invitrogen) labeled with fluorescein was added. Finally, 4, 6-diamidino-2-phenylindole (DAPI) was used to stain the nucleus. The slides were examined by confocal microscopy (Olympus, Tokyo, Japan) at wavelengths of 488 and $568 \mathrm{~nm}$.

\section{Western blot analysis}

Liver tissues were thawed in lysis buffer (Beyotime Institute of Biotechnology), and total protein was extracted. The proteins ( $30 \mu \mathrm{g}$ each) were heated at $100 \stackrel{\circ}{\circ} \mathrm{C}$ for $5 \mathrm{~min}$, then separated by $10 \%$ sodium dodecyl sulfate-polyacrylamide gel electrophoresis (Beyotime Institute of Biotechnology, Shanghai, China), and electrically transferred onto polyvinylidene difluoride membranes (Immobilon-p, Darmstadt, Germany). The membrane was blocked with 5\% BSA in TBST (10 mM Tris- $\mathrm{HCl}, \mathrm{pH} 7.5,150 \mathrm{mM} \mathrm{NaCl}$, and $0.05 \%$ Tween-20) for $1.5 \mathrm{~h}$ at room temperature and then incubated with the primary antibody overnight at $4{ }^{\circ} \mathrm{C}$. The antibodies were purchased from Abcam or Santa Cruz. Next, the membrane was washed and incubated with the horseradish peroxidase-conjugated secondary antibody (1:2000, Biosharp, Hefei, China) for $1 \mathrm{~h}$ at room temperature, respectively. Signals were detected using chemiluminescence with an ECL kit (Advansta, Menlo Park, CA, USA). All bands were analyzed using Image Lab software (version 4.1, Bio-Rad, Hercules, CA, USA).

RNA preparation and reverse transcription-polymerase chain reaction (RT-PCR) analysis

The total RNA from the different groups was extracted using TRIzol Reagent (Invitrogen). The first strand cDNA synthesis was performed using a Revert Aid Synthesis Kit (Thermo Scientific, Rockford, IL, USA), according to the manufacturer's protocol. The mRNAlevels were quantified using aSYBR Premix Ex Kit(Takara Biotech Co. Ltd., Dalian, China) with an ABI PRISM 7500 Detector (Applied Biosystems, Life Technologies, USA). The primer sequences for PCR were as follows: $\beta$-actin (sense, 5'-CGTAAAGACCTCTATGCCAACA-3'; antisense: 5'-GGAGGAGCAATGATCTTGATCT-3'), rat Col1A1 (sense: 5'-GTACATCAGCCCAAACCCCAAG-3'; antisense: 5'-CGGAACCTTCGCTTCCATACTC-3'); mouse Sestrin 2 (sense: 5'-CCTTCTCCACACCCAGACAT3'; antisense: 5'-TATAATCCTGGGCACGGAAG-3'); rat Sestrin 2 (sense: 5'-TTGTGTTTGGCTGTGGGATA3'; antisense: 5'-CCGAGTTGTTCAATGGGTCT-3'); $\alpha$-SMA (sense: 5 '-GTGCTGTCCCTCTATGCCTCTGG- 
3'; antisense: 5'-GGCACGTTGTGAGTCACACCATC-3'), IL-1 $\beta$ (sense: 5'-ATTGTGGCTGTGGAGAAG-3'; antisense: 5'- AAGATGAAGGAAAAGAAGGTG-3'), CCL2 (sense: 5'- ACGTGTTGGCTCAGCCAGA-3'; antisense: 5'- ACTACAGCTTCTTTGGGACACC-3'), TGF- $\beta$ (sense: 5'-GACTCTCCACCTGCAAGACC-3'; antisense: 5'- GGACTGGCGAGCCTTAGTTT-3'), TNF- $\alpha$ (sense: 5'- ATGGCCTCCCTCTCATCAGT-3'; antisense: 5'TTTGCTACGACGTGGGCTAC-3'), mouse Col1A1 (sense: 5'- TTCTCCTGGCAAAGACGGAC-3'; antisense: 5 '-CGGCCACCATCTTGAGACTT -3'). The relative gene expression levels were normalized to $\beta$-actin and calculated using the $2^{-\Delta \triangle \mathrm{Ct}}$ method. RT-PCR was performed in three independent experiments.

\section{Statistical analysis}

All data were presented as the mean \pm standard deviation. Statistical analyses were performed using SPSS software for Windows (version 19; SPSS, Inc., Chicago, IL, USA). One-way analysis of variance and the least significant difference test were used to determine the statistical differences for multiple group comparisons. Values of $P<0.05$ were considered to be statistically significant.

\section{Results}

Sestrin 2 is downregulated in TGF- $\beta 1$-induced HSCs

Activation of HSCs is regarded as a critical step in the development of liver fibrosis. Thus, we used HSC-T6 cells, a rat HSC line, to analyze the expression of Sestrin 2. In our experiment, HSC-T6 cells were challenged with TGF- $\beta 1(10 \mathrm{ng} / \mathrm{mL})$, and Sestrin 2 levels were detected at $48 \mathrm{~h}$. Compared with the control group, Sestrin 2 protein and mRNA levels were decreased by $60 \%$ and $90 \%$, respectively (Fig. $1 \mathrm{~A}$ and B). In contrast, the expression of Col1A1 mRNA was increased by 20 -fold (Fig. 1C). Immunofluorescence analysis revealed high expression of $\alpha$-SMA, a marker of HSC activation (Fig. 1D). Concomitantly, the expression of Sestrin 2 was decreased in the TGF- $\beta$-induced group, suggesting that Sestrin 2 was blocked during the activation of HSC-T6 cells in response to TGF- $\beta 1$.

\section{Sestrin 2 is downregulated in liver fibrosis}

To determine whether Sestrin 2 expression is altered in liver fibrosis, we used the murine model of liver fibrosis induced by $\mathrm{CCl}_{4}$ in order to assess Sestrin 2 expression. The degree of liver fibrosis was evaluated by H\&E and Masson's trichrome staining, respectively (Fig. 2A). Our results showed that the mRNA transcripts of Col1A1 and $\alpha$-SMA in the experimental model of murine liver fibrosis were increased relative to those in mice injected with olive oil (Fig. 2C), while the protein expression of Sestrin 2 was downregulated (Fig. 2B). Therefore, Sestrin 2 protein and mRNA expression was suppressed along with the increased expression of Col1A1 and $\alpha$-SMA mRNA during hepatic fibrogenesis.

\section{Sestrin 2 inhibits the expression of markers of fibrosis in vitro}

To study the effects of Sestrin 2 overexpression in HSC-T6 cells, Sestrin 2 was overexpressed in HSC-T6 cells by transfection. Delivery of Sestrin 2 led to an increase of Sestrin 2 expression, compared with the control group (Fig. 3A and B). HSC-T6 cells transfected with Sestrin 2 exhibited decreased mRNA levels of Col1A1 and $\alpha$-SMA, relative to the control HSC-T6 cells (Fig. 3C and D). Moreover, cell proliferation was downregulated in HSC-T6 cells transfected with the plasmid overexpressing Sestrin 2, compared with the control HSC-T6 cells (Fig. 3E). Immunofluorescence staining of $\alpha$-SMA confirmed reduced levels of positive fluorescence for this marker of HSC activation (Fig. 3F). The protein level of tissue inhibitor of metalloproteinase-1 (TIMP-1) was also reduced due to the transfection of Sestrin 2 (Fig. 3G). Altogether, our findings reveal that Sestrin 2 upregulation is closely related to the expression of markers of fibrosis, including $\alpha$-SMA, Col1A1, and TIMP-1, as well as cell proliferation. 
A

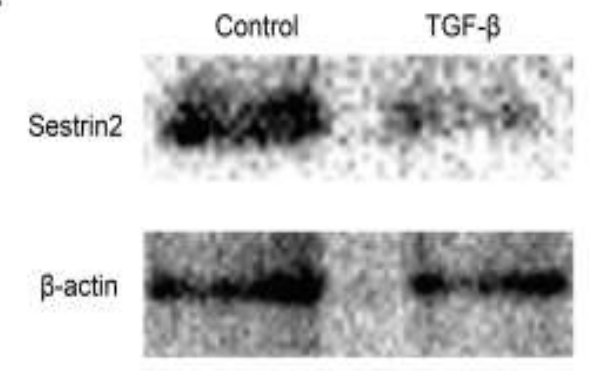

B

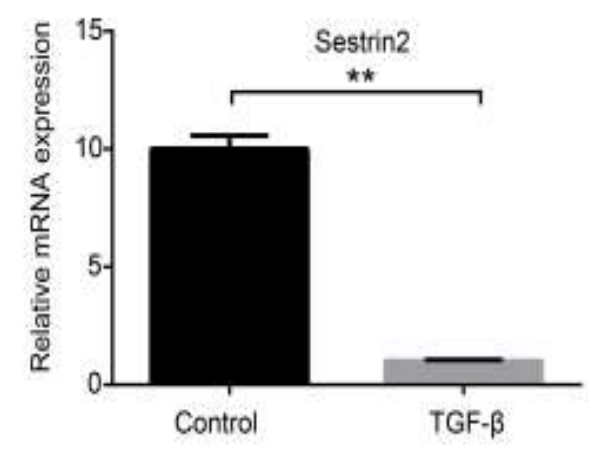

D
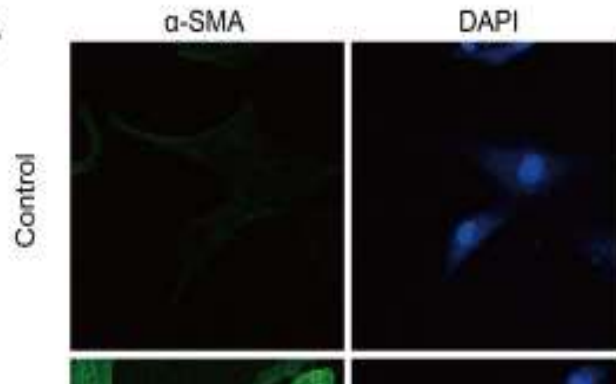

C
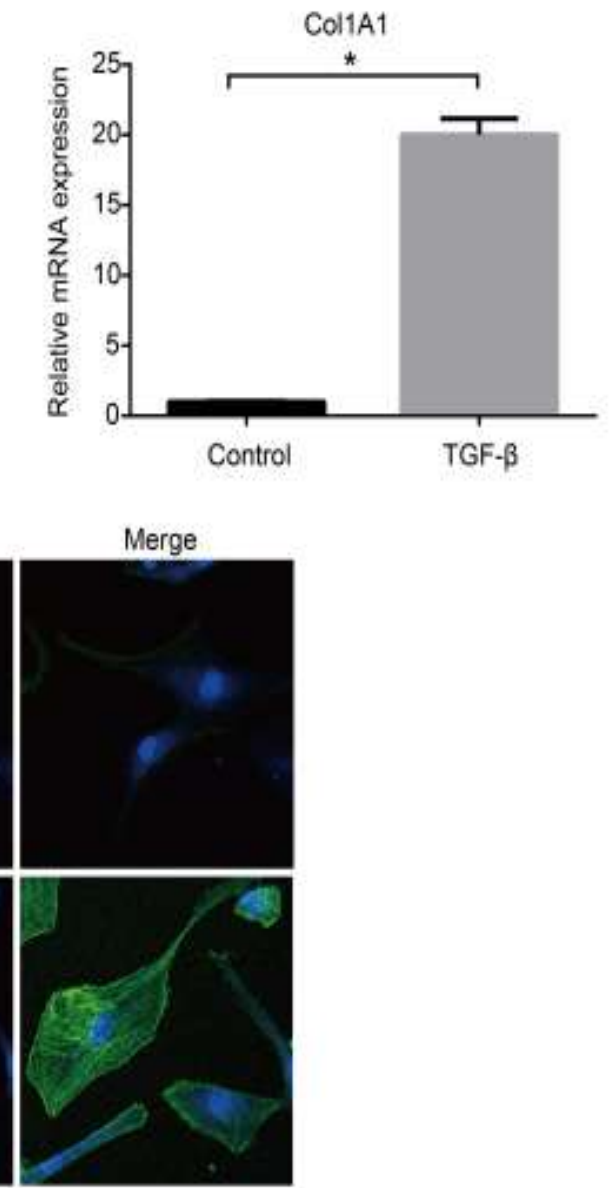

Fig. 1. Relative Sestrin 2 expression in activated HSC-T6 cells. (A) The protein expression of Sestrin 2 was detected in activated HSC-T6 cells by western blotting. (B) The mRNA expression of Sestrin 2 was analyzed by RT-PCR. (C) The mRNA expression of Col1A1 was analyzed by RT-PCR. (D) The protein expression of $\alpha$-SMA (green) was analyzed by immunofluorescence. DAPI (blue) represents cell nuclei $(n=3)$. Data are shown as means \pm standard deviation. ${ }^{*} \mathrm{P}<0.05$.

Sestrin 2 suppresses liver fibrosis in vivo

Next, we explored the role the Sestrin 2 during liver fibrogenesis in vivo. For this purpose, Lenti-Sestrin 2 was transferred intravenously into mice as described in the Materials and Methods section. After $\mathrm{CCl}_{4}$ injections for 4 weeks, serum markers of liver damage were assessed using biochemistry. Quantification of hepatic ALT and AST content demonstrated

\section{KARGER}




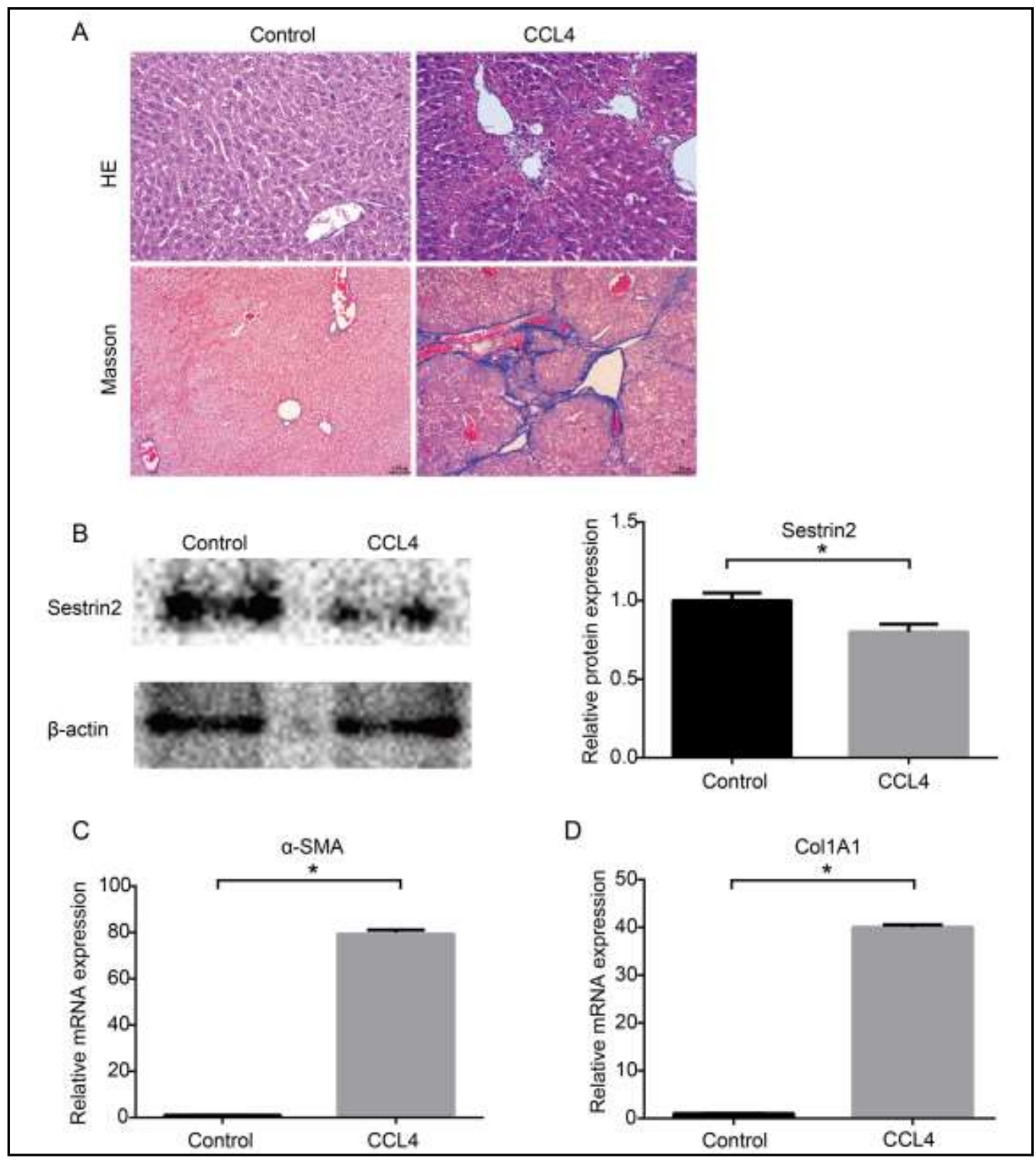

Fig. 2. Relative Sestrin 2 expression in the $\mathrm{CCl}_{4}$-induced mouse model. (A) The degree of liver fibrosis was assessed by H\&E staining and Masson's trichrome staining (200× magnification). (B) The protein expression of Sestrin 2 was detected by western blotting. (C) The mRNA expression of $\alpha$-SMA was detected by RTPCR. (D) The mRNA expression of Col1A1 was detected by RT-PCR. Data are shown as means \pm standard deviation. ${ }^{*} \mathrm{P}<0.05$.

amelioration of liver fibrosis after Lenti-Sestrin 2 treatment (Fig. 4B and C). The mRNA levels of Col1A1 and TGF- $\beta$ were decreased when Sestrin 2 expression was increased (Fig. $4 \mathrm{D})$. Moreover, the mRNA levels of the proinflammatory cytokines TNF- $\alpha$, IL-1 $\beta$, and CCL2 were inhibited due to Sestrin 2 expression (Fig. 4E). In addition, overexpression of Sestrin 2 decreased the severity of inflammation and deposition of collagen, as determined by H\&E and Masson's trichrome staining (Fig. 4F). Increased Sestrin 2 expression also resulted in a reduction of Col1A1 and $\alpha$-SMA expression (Fig. $4 \mathrm{~F}$ and G). Taken together, our results suggest that overexpression of Sestrin 2 can ameliorate the progression of liver fibrosis in vivo.

\section{KARGER}




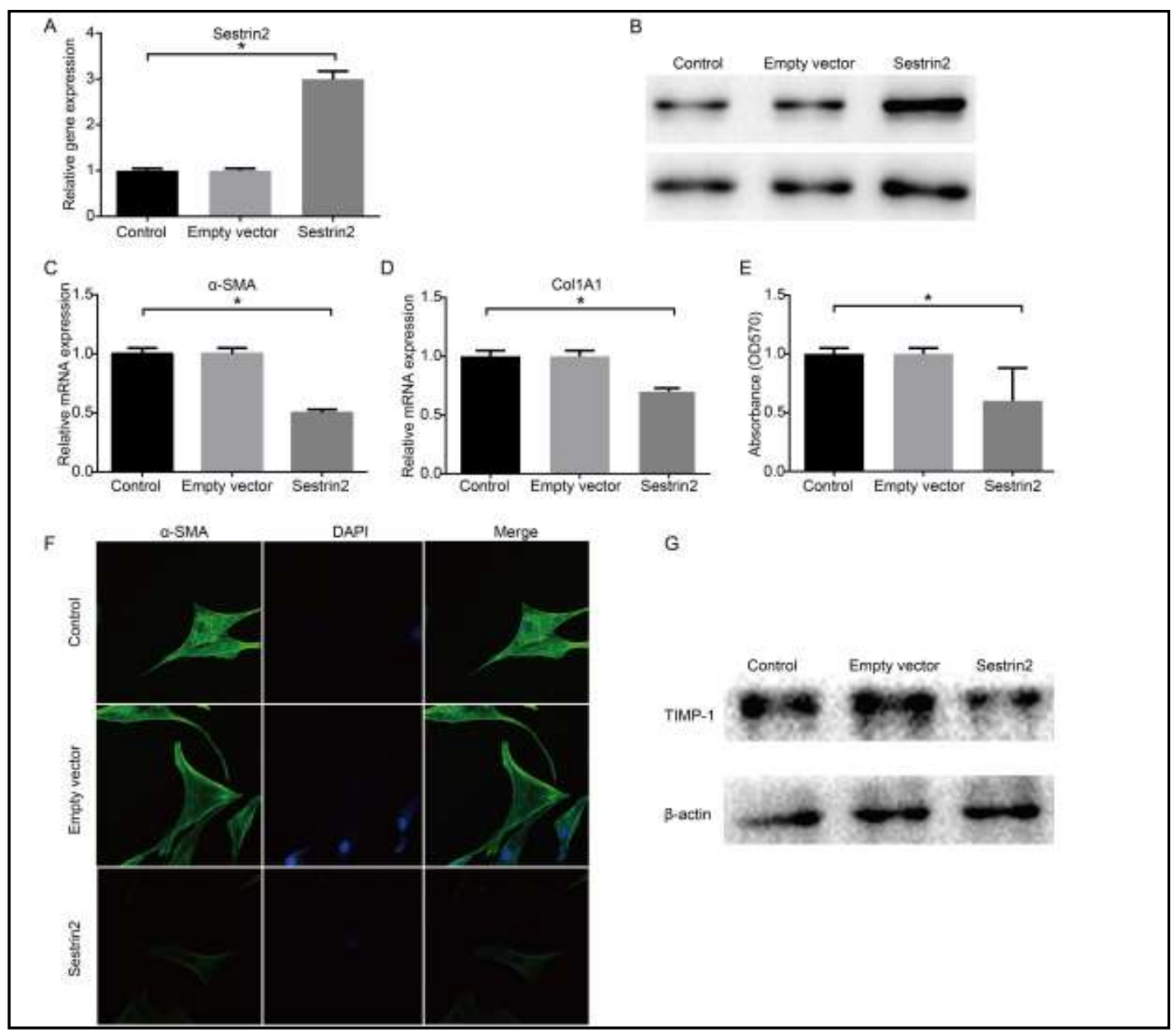

Fig. 3. Sestrin 2 inhibits the expression of markers of fibrosis. (A, B) Analysis of Sestrin 2 mRNA levels by RT-PCR and protein levels by western blot in the control group, empty vector group, and Sestrin 2 group. (C) The mRNA expression of $\alpha$-SMA was evaluated via RT-PCR. (D) The mRNA expression of Col1A1 was evaluated via RT-PCR. (E) The proliferation of cells was measured by the MTT assay. OD, optical density. (F) The $\alpha$-SMA (green) protein level was evaluated by cell staining $(n=3)$. (G) The protein expression of TIMP-1 was evaluated using western blot. Data are shown as means \pm standard deviation. ${ }^{*} \mathrm{P}<0.05$.

Sestrin 2 attenuates the AMPK-mTOR signaling pathway in HSC-T6 cells

We next investigated the potential mechanism by which Sestrin 2 regulates TGF- $\beta$ induced HSC-T6 cell activation. In our experiments, we measured the levels of phosphorylated AMPK, mTOR, and P70S6k by western blotting. We found increased p-AMPK and decreased p-mTOR and p-P70S6k protein expression in the Sestrin 2-overexpressing group, compared with the control group (Fig. 5). Our findings indicate that Sestrin 2 may attenuate the activation of mTOR as an AMPK agonist, thus inhibiting HSC activation.

Sestrin 2 regulates the AMPK-mTOR signaling pathway in experimental liver fibrosis

We further analyzed the relationship between Sestrin 2 and AMPK-mTOR signaling in liver tissues of $\mathrm{CCl}_{4}$-treated mice. Treatment with $\mathrm{CCl}_{4}$ induced mTOR and $\mathrm{P} 70 \mathrm{~S} 6 \mathrm{k}$ phosphorylation but reduced AMPK phosphorylation (Fig. 6). However, Sestrin 2 administration reversed this mechanism. We observed that the phosphorylated mTOR and P70S6k levels were decreased, but the phosphorylated AMPK level was elevated (Fig. 6). Altogether, these results suggest that Sestrin 2 attenuated the development of liver fibrosis through upregulation of AMPK phosphorylation and downregulation of mTOR phosphorylation.

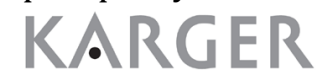




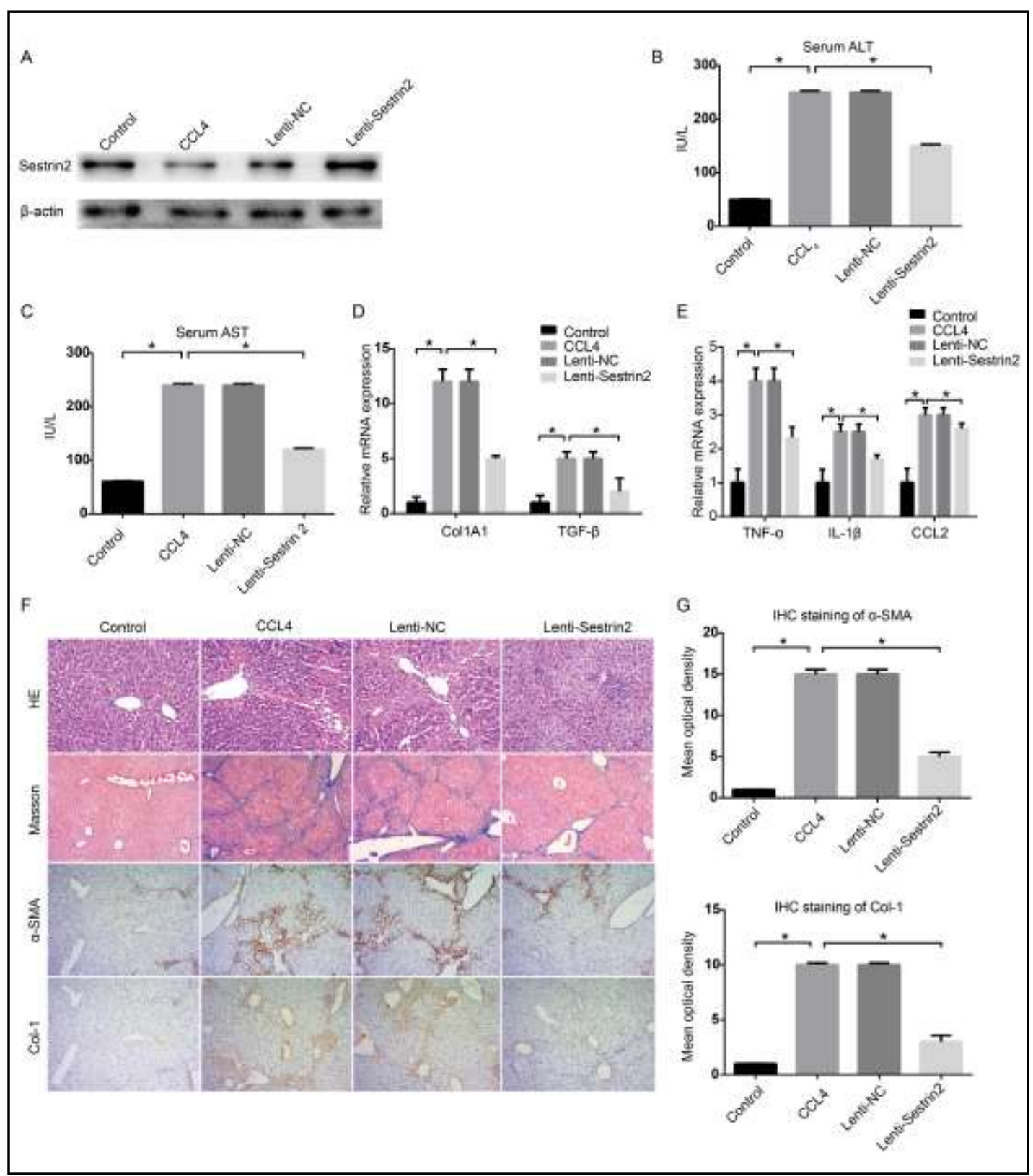

Fig. 4. Sestrin 2 suppresses liver fibrosis in the $\mathrm{CCl}_{4}$-induced mouse model. (A) The protein levels of Sestrin 2 were determined by western blot. (B, C) Serum ALT and AST levels. (D) The mRNA levels of Col1A1 and TGF- $\beta$ were determined by RT-PCR. (E) The mRNA levels of TNF- $\alpha$, IL-1 $\beta$, and CCL2 were determined by RT-PCR. (F, G) Representative images of H\&E and Masson's trichrome staining are shown at 200x magnification. Representative immunohistochemical images of $\alpha$-SMA and Col1A1 staining are shown at $100 \times$ magnification. Data are shown as means \pm standard deviation. ${ }^{*} \mathrm{P}<0.05$. 


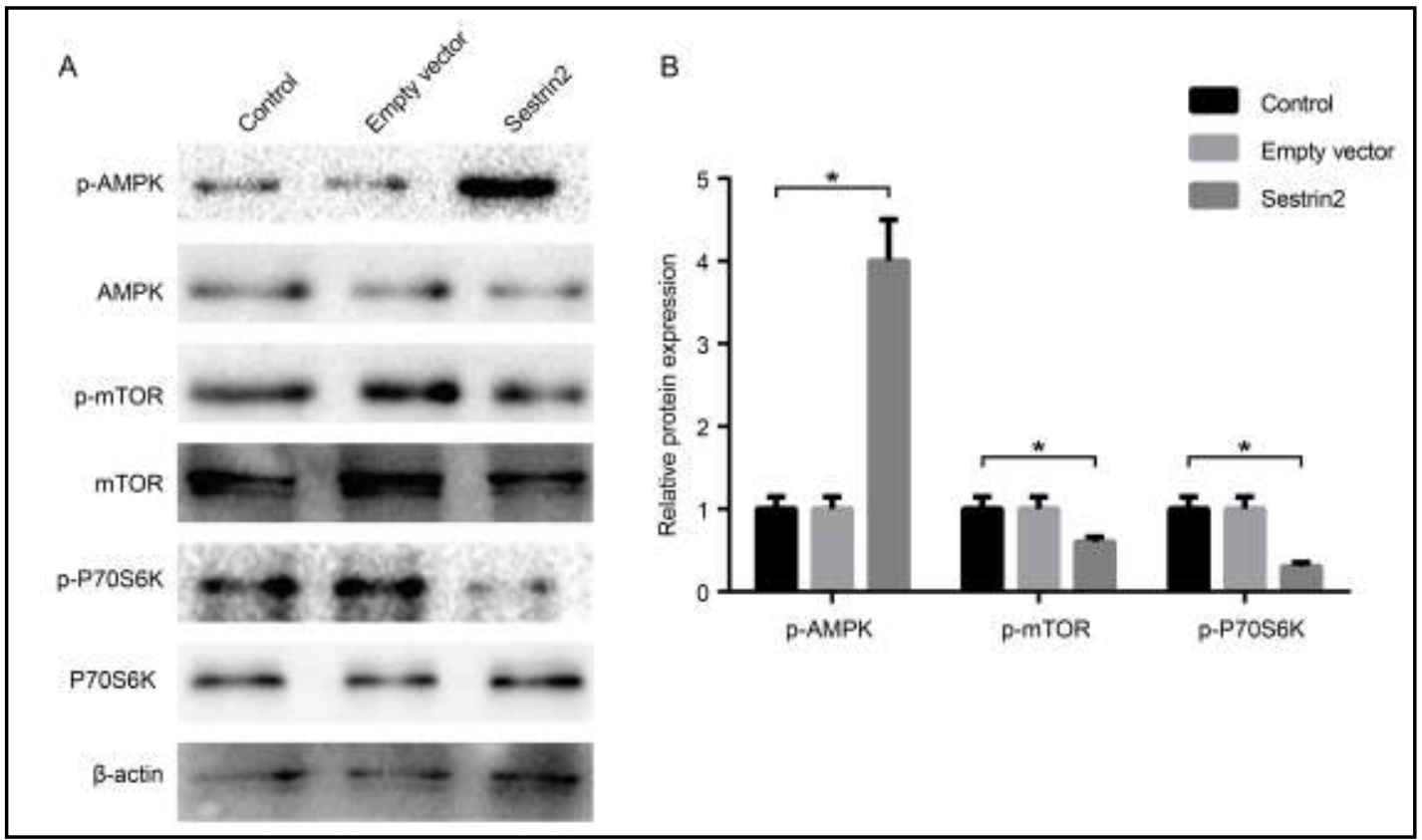

Fig. 5. Effect of Sestrin 2 on the AMPK-mTOR signaling pathway in HSC-T6 cells. The protein expression levels of total phosphorylated AMPK, AMPK, total phosphorylated mTOR, mTOR, total phosphorylated P70S6k, and P70S6k were determined by western blotting.

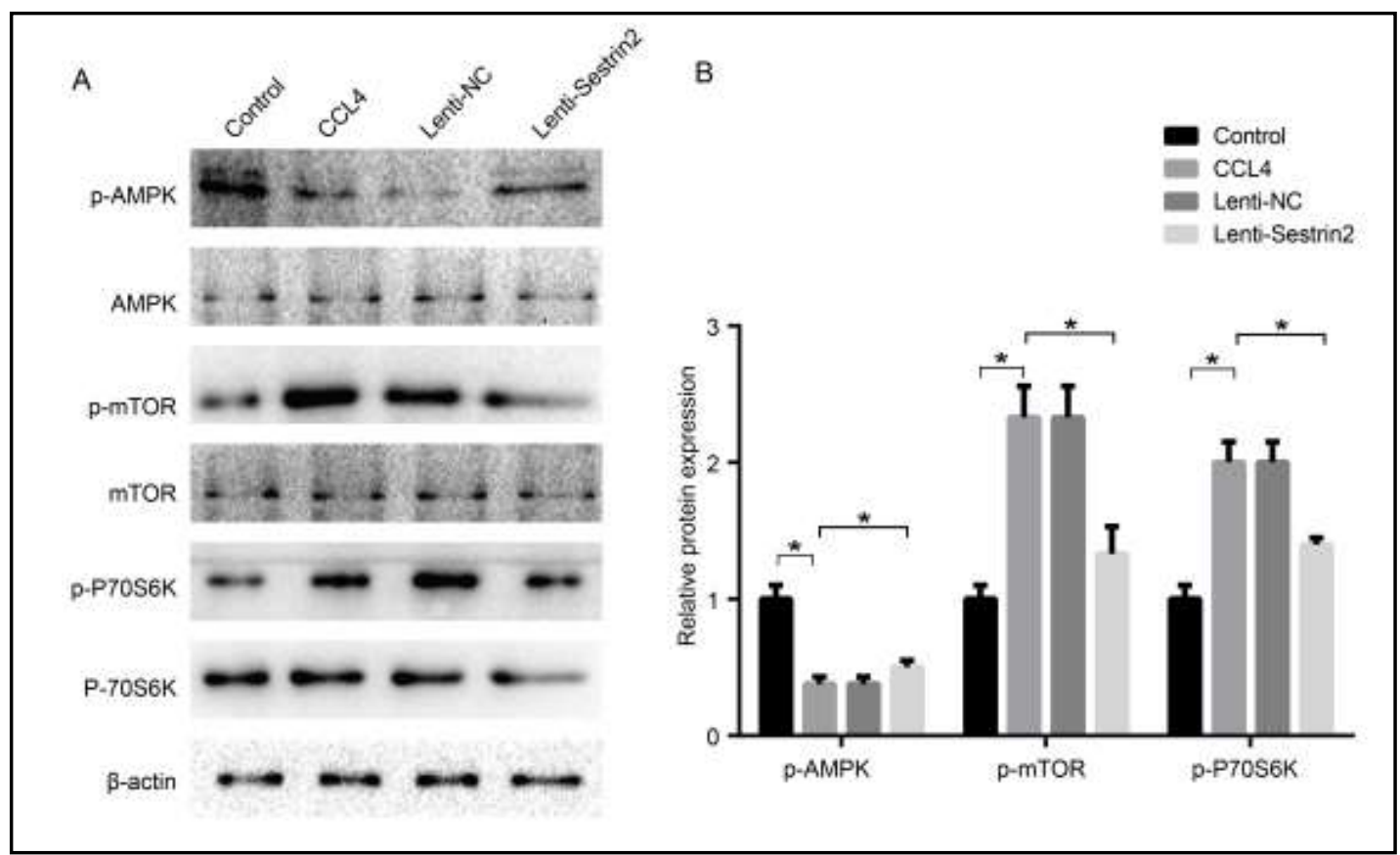

Fig. 6. Effect of Sestrin 2 on the AMPK-mTOR signaling pathway in the $\mathrm{CCl}_{4}$-induced mouse model. The protein expression levels of total phosphorylated AMPK, AMPK, total phosphorylated mTOR, mTOR, total phosphorylated P70S6k, and P70S6k were determined by western blotting. 


\section{Cellular Physiology Cell Physiol Biochem 2018;51:2111-2122

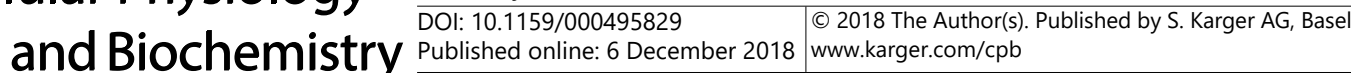 \\ Hu et al.: Sestrin 2 Attenuates Liver Fibrogenesis}

\section{Discussion}

Sestrin 2 is a pivotal repressor of oxidative stress; thus, the function of Sestrin 2 as a therapeutic target for various diseases needs to be thoroughly investigated. Recent evidence has shown that Sestrin 2 is associated with the pathophysiology of several liver diseases. However, to date, the contribution of Sestrin 2 to hepatic fibrogenesis remains unknown. In the present study, we aimed to investigate the role of Sestrin 2 in the development of liver fibrosis and HSC activation. In hypoxic-ischemic encephalopathy, Sestrin 2 has been shown to reverse neurological dysfunction and provide a neuroprotective effect [18]. Additional evidence has indicated that Sestrin 2 upregulation protects against angiotensin II-induced endothelial dysfunction [19]. Our results unveiled that administration of Sestrin 2 ameliorates liver fibrosis in vivo. In addition, we found that Sestrin 2 was inversely correlated with the degree of HSC activation. These results are concomitant with previous findings in non-small cell lung cancer [15].

Recently, Kim et al. [20] have suggested that Sestrin 2 reduces endotoxin-derived injury via toll-like receptor ligands. Moreover, Hu et al. [10] have demonstrated that knockdown of Sestrin 2 enhances apoptotic cell death in macrophages. Furthermore, overexpression of Sestrin 2 may improve radiation-induced myocardial damage [21]. Therefore, the role of Sestrin 2 seems ubiquitous and complex, a phenomenon that requires further investigation. First, we found that Sestrin 2 expression is suppressed in activated HSCs. This mechanism mimics the experimental model of murine liver fibrosis in which hepatic fibrogenesis inversely correlates with Sestrin 2 expression. Next, we found that overexpression of Sestrin 2 inhibited HSC activation and the degree of liver fibrosis. The histopathological examination revealed that the severity of liver fibrosis was alleviated by induction of Sestrin 2 expression. Accordingly, increased expression of serum markers of liver damage, including ALT and AST, were attenuated. Additionally, cell proliferation of HSCs was modulated. Moreover, we performed a scratch test to evaluate migration and found that overexpression of Sestrin2 decreased the migration of HSCs (results not shown).

Next, we tested the potential mechanism by which Sestrin 2 inhibited the effects on HSC activation. Sestrin 2 acts as an inhibitor of mTOR by upregulating the activation of AMPK [22]. Interestingly, Sestrin 2-knockout mice fed a high-fat diet displayed mTOR-S6K inactivation [9]. Sestrin 2 is a pivotal modulator of AMPK, and, recently, the role of Sestrin 2 in the mTOR signaling cascade has been partly unveiled $[23,24]$. A recent finding revealed that liver fibrosis can be ameliorated mainly through phosphorylation of AMPK and blockade of mTOR-dependent cascades [25]. Taken together, we aimed to explore whether Sestrin 2 regulates cell proliferation via modulation of the AMPK and mTOR signaling pathways. Using western blot analysis, we showed that overexpression of Sestrin 2 led to increased p-AMPK protein expression but decreased p-mTOR protein expression both in vivo and in vitro. These data support the notion that AMPK and mTOR may be involved in HSC activation and hepatic fibrogenesis.

There are some deficiencies in this study that should be noted. First, hepatocytes are the major cell type in the liver. In the present study, the expression of Sestrin 2 in hepatocytes was not detected. Second, the role of Sestrin 2 in liver pathophysiology is complex, but we only focused on liver fibrosis in this study.

\section{Conclusion}

Collectively, our results indicate that Sestrin 2 can ameliorate $\mathrm{CCl}_{4}$-induced liver fibrosis. Our data also suggest that overexpression of Sestrin 2 can modulate HSC activation, which is very likely associated with the AMPK and m-TOR signaling pathways. Since Sestrin 2 is a critical component of liver pathophysiology, it might be worth investigating the underlying beneficial roles of Sestrin 2 for the treatment of chronic liver disease. 


\section{Cellular Physiology Cell Physiol Biochem 2018;51:2111-2122

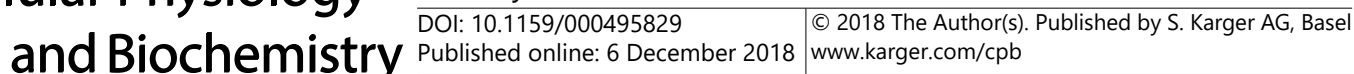

Hu et al.: Sestrin 2 Attenuates Liver Fibrogenesis

\section{Acknowledgements}

This work was supported by The Natural Science Foundation of Zhejiang Province (grant no. LY14H030010).

\section{Disclosure Statement}

The authors declare that they have no conflicts of interest.

\section{References}

1 Bataller R, Brenner DA: Liver fibrosis. J Clin Invest 2005;115:209-218.

-2 Ellis EL, Mann DA: Clinical evidence for the regression of liver fibrosis. J Hepatol 2012;56:1171-1180.

-3 Hellerbrand C: Molecular targets for antifibrotic therapy in liver disease: using magic bullets for crossfire rather than a one-sided shotgun attack. Gut 2014;63:1039-1041.

-4 Friedman SL: Mechanisms of hepatic fibrogenesis. Gastroenterology 2008;134:1655-1669. Puche JE, Saiman Y, Friedman SL: Hepatic stellate cells and liver fibrosis. Compr Physiol 2013;3:1473-1492. Trautwein C, Friedman SL, Schuppan D, Pinzani M: Hepatic fibrosis: Concept to treatment. J Hepatol 2015;62:S15-24.

7 Lee JH, Budanov AV, Karin M: Sestrins orchestrate cellular metabolism to attenuate aging. Cell Metab 2013;18:792-801.

8 Budanov AV, Lee JH, Karin M: Stressin' Sestrins take an aging fight. EMBO Mol Med 2010;2:388-400.

-9 Lee JH, Budanov AV, Talukdar S, Park EJ, Park HL, Park HW, Bandyopadhyay G, Li N, Aghajan M, Jang I, Wolfe AM, Perkins GA, Ellisman MH, Bier E, Scadeng M, Foretz M, Viollet B, Olefsky J, Karin M: Maintenance of metabolic homeostasis by Sestrin2 and Sestrin3. Cell Metab 2012;16:311-321.

10 Hu HJ, Shi ZY, Lin XL, Chen SM, Wang QY, Tang SY: Upregulation of Sestrin2 expression protects against macrophage apoptosis induced by oxidized low-density lipoprotein. DNA Cell Biol 2015;34:296-302.

11 Budanov AV, Shoshani T, Faerman A, Zelin E, Kamer I, Kalinski H, Gorodin S, Fishman A, Chajut A, Einat P, Skaliter R, Gudkov AV, Chumakov PM, Feinstein E: Identification of a novel stress-responsive gene Hi95 involved in regulation of cell viability. Oncogene 2002;21:6017-6031.

-12 Chantranupong L, Wolfson RL, Orozco JM, Saxton RA, Scaria SM, Bar-Peled L, Spooner E, Isasa M, Gygi SP, Sabatini DM: The Sestrins interact with GATOR2 to negatively regulate the amino-acid-sensing pathway upstream of mTORC1. Cell Rep 2014;9:1-8.

13 Wang N, Pan W, Zhu M, Zhang M, Hao X, Liang G, Feng Y: Fangchinoline induces autophagic cell death via p53/sestrin2/AMPK signalling in human hepatocellular carcinoma cells. Br J Pharmacol 2011;164:731742.

14 Lee JH, Budanov AV, Park EJ, Birse R, Kim TE, Perkins GA, Ocorr K, Ellisman MH, Bodmer R, Bier E, Karin M: Sestrin as a feedback inhibitor of TOR that prevents age-related pathologies. Science 2010;327:1223-1228.

15 Chen KB, Xuan Y, Shi WJ, Chi F, Xing R, Zeng YC: Sestrin2 expression is a favorable prognostic factor in patients with non-small cell lung cancer. Am J Transl Res 2016;8:1903-1909.

16 Park HW, Park H, Ro SH, Jang I, Semple IA, Kim DN, Kim M, Nam M, Zhang D, Yin L, Lee JH: Hepatoprotective role of Sestrin2 against chronic ER stress. Nat Commun 2014;5:4233.

17 Parmigiani A, Nourbakhsh A, Ding B, Wang W, Kim YC, Akopiants K, Guan KL, Karin M, Budanov AV: Sestrins inhibit mTORC1 kinase activation through the GATOR complex. Cell Rep 2014;9:1281-1291.

18 Shi X, Xu L, Doycheva DM, Tang J, Yan M, Zhang JH: Sestrin2, as a negative feedback regulator of mTOR, provides neuroprotection by activation AMPK phosphorylation in neonatal hypoxic-ischemic encephalopathy in rat pups. J Cereb Blood Flow Metab 2017;37:1447-1460.

19 Yi L, Li F, Yong Y, Jianting D, Liting Z, Xuansheng H, Fei L, Jiewen L: Upregulation of sestrin-2 expression protects against endothelial toxicity of angiotensin II. Cell Biol Toxicol 2014;30:147-156.

20 Kim MG, Yang JH, Kim KM, Jang CH, Jung JY, Cho IJ, Shin SM, Ki SH: Regulation of Toll-like receptor-mediated Sestrin2 induction by AP-1, Nrf2, and the ubiquitin-proteasome system in macrophages. Toxicol Sci 2015;144:425-435. 


\section{Cellular Physiology Cell Physiol Biochem 2018;51:2111-2122 \begin{tabular}{ll|l} 
and Biochemistry & Dublished online: 6 December 2018 & $\begin{array}{l}\text { O } 2018 \text { The Author(s). Published by S. Karger AG, Basel } \\
\text { www.karger.com/cpb }\end{array}$ \\
\cline { 2 - 3 }
\end{tabular}}

Hu et al.: Sestrin 2 Attenuates Liver Fibrogenesis

21 Zeng YC, Chi F, Xing R, Zeng J, Gao S, Chen JJ, Wang HM, Duan QY, Sun YN, Niu N, Tang MY, Wu R: Sestrin2 protects the myocardium against radiation-induced damage. Radiat Environ Biophys 2016;55:195-202.

-22 Kim GT, Lee SH, Kim YM: Quercetin Regulates Sestrin 2-AMPK-mTOR Signaling Pathway and Induces Apoptosis via Increased Intracellular ROS in HCT116 Colon Cancer Cells. J Cancer Prev 2013;18:264-270.

23 Morrison A, Chen L, Wang J, Zhang M, Yang H, Ma Y, Budanov A, Lee JH, Karin M, Li J: Sestrin2 promotes LKB1-mediated AMPK activation in the ischemic heart. FASEB J 2015;29:408-417.

-24 Peng M, Yin N, Li MO: Sestrins function as guanine nucleotide dissociation inhibitors for Rag GTPases to control mTORC1 signaling. Cell 2014;159:122-133.

25 Wu YL, Zhang YJ, Yao YL, Li ZM, Han X, Lian LH, Zhao YQ Nan JX: Cucurbitacin E ameliorates hepatic fibrosis in vivo and in vitro through activation of AMPK and blocking mTOR-dependent signaling pathway. Toxicol Lett 2016;258:147-158. 